\title{
Cost-Effectiveness of Endovascular Thrombectomy in Childhood Stroke: An Analysis of the Save ChildS Study
}

\author{
Wolfgang G. Kunz, ${ }^{\mathrm{a}, *}$ Peter B. Sporns, ${ }^{\mathrm{b}, \mathrm{c}, *}$ Marios N. Psychogios, ${ }^{\mathrm{b}}$ Jens Fiehler, $^{\mathrm{c}}$ René Chapot, ${ }^{\mathrm{d}}$ \\ Franziska Dorn, ${ }^{\mathrm{e}}$ Astrid Grams, ${ }^{\mathrm{f}}$ Andrea Morotti, ${ }^{\mathrm{g}}$ Patricia Musolino, ${ }^{\mathrm{h}}$ Sarah Lee, ${ }^{\mathrm{i}}$ André Kemmling, \\ Hans Henkes, ${ }^{\mathrm{k}}$ Omid Nikoubashman, ${ }^{\mathrm{l}}$ Martin Wiesmann, ${ }^{\mathrm{l}}$ Ulf Jensen-Kondering, ${ }^{\mathrm{m}}$ \\ Markus Möhlenbruch, ${ }^{\mathrm{n}}$ Marc Schlamann, ${ }^{\mathrm{o}}$ Wolfgang Marik, ${ }^{\mathrm{p}}$ Stefan Schob, ${ }^{\mathrm{q}}$ Christina Wendl, ${ }^{\mathrm{r}}$ \\ Bernd Turowski, ${ }^{\mathrm{s}}$ Friedrich Götz, ${ }^{\mathrm{t}}$ Daniel Kaiser, ${ }^{\mathrm{u}}$ Konstantinos Dimitriadis, ${ }^{\mathrm{v}}$ Alexandra Gersing, ${ }^{\mathrm{w}}$ \\ Thomas Liebig, ${ }^{\mathrm{w}}$ Jens Ricke, ${ }^{\mathrm{a}}$ Paul Reidler, ${ }^{\mathrm{a}}$ Moritz Wildgruber, ${ }^{\mathrm{a}, \dagger}$ Sebastian Mönch, ${ }^{\mathrm{a}, \dagger}$ for the Save \\ ChildS Investigators
}

\author{
${ }^{a}$ Department of Radiology, University Hospital, LMU Munich, Munich, Germany \\ bepartment of Neuroradiology, Clinic for Radiology \& Nuclear Medicine, University Hospital Basel, Switzerland \\ 'Department of Diagnostic and Interventional Neuroradiology, University Medical Center Hamburg-Eppendorf, Hamburg, Germany \\ ${ }^{\mathrm{d}}$ Department of Neuroradiology, Alfried-Krupp Hospital, Essen, Germany \\ ${ }^{e}$ Department of Neuroradiology, University Hospital Bonn, Bonn, Germany \\ fDepartment of Neuroradiology, Medical University of Innsbruck, Innsbruck, Austria. \\ ${ }^{9}$ Neurology Unit, Department of Clinical and Experimental Sciences, University of Brescia, Brescia, Italy \\ ${ }^{h}$ Department of Neurology, Massachusetts General Hospital, Harvard Medical School, Boston, MA, USA \\ 'Division of Child Neurology, Department of Neurology, Stanford University, Stanford, CA, USA \\ 'Department for Neuroradiology, University Hospital Marburg, Marburg, Germany \\ ${ }^{k}$ Department of Neuroradiology, Klinikum Stuttgart, Stuttgart, Germany \\ 'Department of Neuroradiology, Aachen University, Aachen, Germany \\ mepartment of Radiology and Neuroradiology, University Hospital of Schleswig-Holstein, Kiel, Germany \\ ${ }^{n}$ Department of Neuroradiology, Heidelberg University Hospital, Heidelberg, Germany \\ ${ }^{\circ}$ Department of Neuroradiology, University Hospital of Cologne, Cologne, Germany \\ ${ }^{p}$ Division of Neuroradiology and Musculoskeletal Radiology, Department of Biomedical Imaging and Image-guided Therapy, Medical University \\ of Vienna, Vienna, Austria \\ ${ }^{9}$ Department for Neuroradiology, University Hospital Leipzig, Leipzig, Germany \\ 'Department of Radiology, University Hospital Regensburg, Regensburg, Germany \\ Institute of Neuroradiology, University Hospital Duesseldorf, Duesseldorf, Germany \\ tDepartment of Diagnostic and Interventional Neuroradiology, Hannover Medical School, Hannover, Germany \\ uDepartment of Neuroradiology, University Hospital Carl Gustav Carus, Dresden, Germany \\ 'Department of Neurology, University Hospital, LMU Munich, Munich, Germany \\ winstitute of Diagnostic and Interventional Neuroradiology, University Hospital, LMU Munich, Munich, Germany
}

Background and Purpose The Save ChildS Study demonstrated that endovascular thrombectomy (EVT) is a safe treatment option for pediatric stroke patients with large vessel occlusions (LVOs) with high recanalization rates. Our aim was to determine the long-term cost, health consequences and cost-effectiveness of EVT in this patient population.

Methods In this retrospective study, a decision-analytic Markov model estimated lifetime costs and quality-adjusted life years (QALYs). Early outcome parameters were based on the entire Save ChildS Study to model the EVT group. As no randomized data exist, the Save ChildS patient subgroup with unsuccessful recanalization was used to model the standard of care group. For modeling of lifetime estimates, pediatric and adult input parameters were obtained from the current lit-
Correspondence: Wolfgang G. Kunz Department of Radiology, University Hospital, LMU Munich, Marchioninistr 15,81377 Munich, Germany Tel: $+49-89-4400-73630$ Fax: +49-89-4400-78832 E-mail:wolfgang.kunz@med.Imu.de https://orcid.org/0000-0002-5021-1952

Received: May 4, 2021

Revised: September 21, 2021

Accepted: September 23, 2021 
erature. The analysis was conducted in a United States setting applying healthcare and societal perspectives. Probabilistic sensitivity analyses were performed. The willingness-to-pay threshold was set to $\$ 100,000$ per QALY.

Results The model results yielded EVT as the dominant (cost-effective as well as cost-saving) strategy for pediatric stroke patients. The incremental effectiveness for the average age of 11.3 years at first stroke in the Save ChildS Study was determined as an additional 4.02 lifetime OALYS, with lifetime cost-savings that amounted to $\$ 169,982$ from a healthcare perspective and $\$ 254,110$ when applying a societal perspective. Acceptability rates for EVT were $96.60 \%$ and $96.66 \%$ for the healthcare and societal perspectives.

Conclusions EVT for pediatric stroke patients with LVOs resulted in added OALY and reduced lifetime costs. Based on the available data in the Save ChildS Study, EVT is very likely to be a cost-effective treatment strategy for childhood stroke.

Keywords Pediatrics; Stroke; Thrombectomy; Cost-benefit analysis
*These authors contributed equally to the manuscript as first author. ${ }^{\dagger}$ These authors contributed equally to the manuscript as last author.

\section{Introduction}

Ischemic stroke ranks among the most cost-intensive diseases in all Western countries. Its currently estimated direct and indirect annual costs in the United States are $\$ 50$ billion. ${ }^{1}$ For patients with acute anterior circulation large vessel occlusions (LVO), randomized controlled trials (RCTs) established endovascular thrombectomy (EVT) as standard of care (SC) for adults. ${ }^{2}$ The widespread adoption has been supported and accelerated by several economic analyses, demonstrating EVT to be longterm cost-saving for healthcare systems across many different countries. $^{3-7}$

Pediatric arterial ischemic stroke differs vastly from adult stroke in prevalence, etiology, risk factors, symptoms, and neurologic outcome. ${ }^{8}$ The SC is largely based on supportive care. The evidence on efficacy and safety of intravenous thrombolysis remains scarce after the Thrombolysis in Pediatric Stroke (TIPS) trial was closed for lack of accrual. ${ }^{9,10}$ In recent years, severe strokes in children with LVO are increasingly being treated with EVT in specialized centers. ${ }^{11}$ The Save ChildS Study demonstrated that EVT is a safe and effective treatment option for pediatric stroke patients and that a favorable outcome similar to EVT in adults can be achieved. ${ }^{12}$

Pediatric patients represent a demographic group with a low incidence of stroke yet suffer the longest consequences of potential disability. Pediatric stroke is thereby linked to substantial direct healthcare costs in the first year, yet few reports exist on the long-term care costs. ${ }^{13}$ Given the longer remaining lifespan in children, pediatric stroke also influences indirect costs, e.g., as a result of reduced lifetime productivity and need for informal care by next of kin. ${ }^{14}$

Our study aim was to determine the long-term cost and health consequences as well as cost-effectiveness of EVT in this patient population.

\section{Methods}

\section{Ethics statement}

The present study complies with the principles of the Declaration of Helsinki (1964). The present study was approved by the Institutional Review Board, and the requirement for written informed consent was waived. All of our patient population was previously reported in the Save ChildS Study. ${ }^{12}$

\section{Model structure}

A decision model using dedicated analytic software (TreeAge Pro 2021 version 21.1.0, TreeAge, Williamstown, MA, USA) was applied to evaluate the cost-effectiveness of EVT versus SC. Adhering to the recommendations by the Second Panel on Cost-Effectiveness in Health and Medicine. ${ }^{14}$ We performed analyses adopting the healthcare as well as a societal perspective. A short-run model was created to analyze costs and functional outcomes within the initial 90 days after the index stroke. Pediatric stroke patients with LVO enter the model on admission to the hospital, receive either EVT or SC and afterwards enter one of the seven health states according to the degree of disability as assessed by the modified Rankin Scale (mRS).

A long-run Markov state transition model was used to estimate the expected costs and outcomes over the lifetime of the patient, using a cycle length of 1 year. During each cycle, patients could either remain in the same health state, experience a recurrent stroke and recover to the same $\mathrm{mRS}$ state or transit to a lower mRS state, or die from other causes. Absorbing 


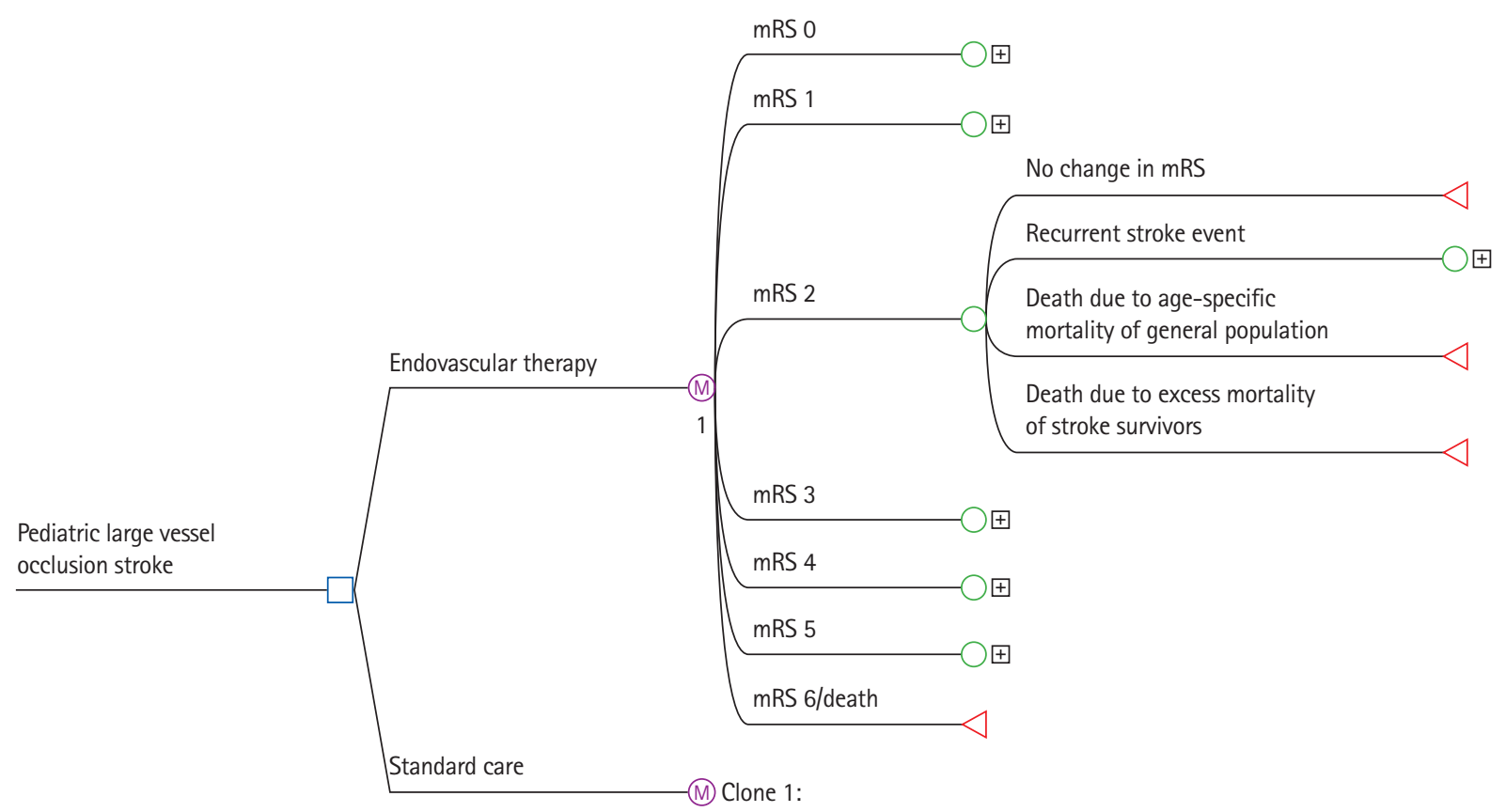

Figure 1. Model structure. mRS, modified Rankin Scale.

states were death due to stroke or death due to any other cause. The model structure is shown in Figure 1.

\section{Model input parameters}

The input parameters for the model were based on the most recently published literature providing the best available level of evidence (Table 1). ${ }^{2,715-37}$ To study the impact of patient age, we based this study on a dedicated systematic and comprehensive literature review regarding age dependency of model input parameters. ${ }^{38}$

\section{Initial and transition probabilities}

The initial probabilities (i.e., the probability of entering a specific mRS health state at hospital discharge) were based on outcomes in the Save ChildS Study. ${ }^{12}$ This multicenter trial included 73 children from 27 participating stroke centers. There is no randomized data on the use of EVT versus SC in children. Following careful interdisciplinary discussion among the study coordinators, the best approach to model outcomes for the SC group was considered to be based on the Save ChildS study subgroup with unsuccessful recanalization (modified Thrombolysis in Cerebral Infarction [mTICl] 0-2a). The outcome parameters of the entire Save ChildS Study population were used to model the EVT group, i.e., any recanalization status ( $\mathrm{mTICl} 0-3$ ). The mRS outcomes for both subgroups are provided in Figure 2.

The transition probabilities consisted of the probability of staying in the same health state, the annual recurrent stroke rate, the probabilities of reentering the same or a lower health state following recurrent stroke, and the age-specific annual death rate. Based on several long-term follow-up studies on functional recovery after stroke, we assumed no significant mRS change in the long-term after stroke. ${ }^{15-17}$ The pediatric recurrent stroke rate was obtained from a long-term follow-up study of childhood stroke. ${ }^{39}$ The adult recurrent stroke rate is known to decrease over time after the index stroke and to increase with patient age; corresponding rates were available from a contemporary long-term study in adults. ${ }^{18}$ Both aspects were built into the model. The age-specific annual death rate was drawn from the United States Life Table. ${ }^{19}$ The death rate was adjusted according to adult hazard rate ratios for each of the mRS health states ${ }_{1}{ }^{20}$ which are considered to be age-independent. ${ }^{20}$ The probabilities to reenter the health states following a recurrent stroke were approximated for both arms using age-dependent resampling of the initial probabilities of the Save ChildS Study and in case of adult recurrent stroke following the according age subgroup receiving SC in a meta-analysis on LVO in adult patients, a conservative assumption based on literature review. ${ }^{18,40-42}$

\section{Direct costs}

Both direct acute and long-term costs were stratified for each of the seven health states (mRS levels 0-6). The acute treatment costs in the first 90 days were based on a study that determined the implied costs for each mRS score in adults. ${ }^{21}$ The 
Table 1. Base-case values and sources of model input parameters

\begin{tabular}{|c|c|c|c|}
\hline Model input & Base-case value $^{*}$ & Distribution & Source \\
\hline \multicolumn{4}{|l|}{ Initial probabilities } \\
\hline $\begin{array}{l}\text { For each health state mRS 0-6 of EVT-treated } \\
\text { patients }\end{array}$ & $\begin{array}{l}\text { Discharge } \mathrm{mRS} \text { distribution for } \mathrm{mTICl} 0-3 \\
\text { recanalization }\end{array}$ & Dirichlet & $\begin{array}{l}\text { Save ChildS Study Sporns et al. } \\
\text { (Figure 2) }\end{array}$ \\
\hline $\begin{array}{l}\text { For each health state mRS 0-6 of SC-treated } \\
\text { patients }\end{array}$ & $\begin{array}{l}\text { Discharge mRS distribution for } \mathrm{mTICl} 0-2 a \\
\text { recanalization }\end{array}$ & Dirichlet & $\begin{array}{l}\text { Save ChildS Study Sporns et al. }{ }^{12} \\
\text { (Figure 2) }\end{array}$ \\
\hline Treatment with IVT & 0.219 & $\beta$ & Save ChildS Study Sporns et al..$^{12}$ \\
\hline \multicolumn{4}{|l|}{ Transition probabilities } \\
\hline Pediatric recurrent stroke rate & 0.066 (over first 5 years) & $\beta$ & Sträter et al. ${ }^{39}$ \\
\hline Adult recurrent stroke rate & Based on cycle age and time since index stroke & $\beta$ & Pennlert et al. ${ }^{18}$ \\
\hline Annual death rate & 0.000103 (for 11 years old) & $\beta$ & US Life Table $2017^{44}$ \\
\hline $\begin{array}{l}\text { Annual death hazard rates for survivors mRS } \\
0 / 1 / 2 / 3 / 4 / 5\end{array}$ & $1.53 / 1.52 / 2.17 / 3.18 / 4.55 / 6.55$ & Log normal & Kunz et al. ${ }^{38}$ \\
\hline After recurrent pediatric stroke & $\begin{array}{l}\text { Discharge } \mathrm{mRS} \text { distribution for } \mathrm{mTICI} 0-2 \mathrm{a} \\
\text { recanalization }\end{array}$ & Dirichlet & Save ChildS Study Sporns et al..$^{12}$ \\
\hline After recurrent adult stroke & 90-day mRS control arm & Dirichlet & HERMES Goyal et al. ${ }^{2}$ \\
\hline \multicolumn{4}{|l|}{ Health care costs } \\
\hline $\begin{array}{l}\text { Costs within first } 90 \text { days after stroke for mRS } \\
\text { 0/1/2/3/4/5/6 (excluding IVT and EVT) }\end{array}$ & $\begin{array}{l}\$ 8,778 / \$ 12,117 / \$ 19,031 / \$ 23,536 / \$ 31,537 / \\
\$ 37,674 / \$ 8,856\end{array}$ & $\gamma$ & Dawson et al. ${ }^{21}$ \\
\hline Additional cost of IVT treatment & $\$ 7,641$ & $\gamma$ & NIS $2018^{43}$ \\
\hline Additional cost of EVT treatment & $\$ 15,977$ & $\gamma$ & Shireman et al. ${ }^{7}$ \\
\hline $\begin{array}{l}\text { Long-term annual costs after stroke for mRS } \\
0 / 1 / 2 / 3 / 4 / 5\end{array}$ & $\begin{array}{l}\$ 12,344 / \$ 12,711 / \$ 14,704 / \$ 25,258 / \$ 51,104 / \\
\$ 75,131\end{array}$ & $\gamma$ & Shireman et al. ${ }^{7}$ \\
\hline Recurrent stroke hospitalization & $\$ 25,283$ & $\gamma$ & Chambers et al. ${ }^{32}$ \\
\hline \multicolumn{4}{|l|}{ Utilities } \\
\hline $\mathrm{mRS} 0 / 1 / 2 / 3 / 4 / 5 / 6$ & 1.00/0.91/0.76/0.65/0.33/0.00/0.00 & $\beta$ & Chaisinanunkul et al. ${ }^{33}$ \\
\hline \multicolumn{4}{|l|}{ Societal costs } \\
\hline \multicolumn{4}{|l|}{ Paid workforce productivity } \\
\hline Average annual earnings of employed population & $\$ 13,441$ (for 18 to 24 years old) & $\gamma$ & US Census Bureau 2018 \\
\hline Population employment rate & 0.400 (for 18 to 19 years old) & $\beta$ & US Bureau of Labor Statistics 2020 \\
\hline Relative earnings of stroke survivors & 0.825 & $\beta$ & Vyas et al. ${ }^{46}$ \\
\hline Return-to-work after stroke mRS 0/1/2/3/4/5 & 0.63/0.72/0.49/0.19/0.14/0.00 & $\beta$ & Tanaka et al. ${ }^{48}$ \\
\hline \multicolumn{4}{|l|}{ Unpaid domestic productivity } \\
\hline Informal annual caregiving costs & $\begin{array}{l}\text { mRS 0-1: } \$ 1,650 \\
\text { mRS 2-5: } \$ 8,253\end{array}$ & $\gamma$ & Hickenbottom et al. ${ }^{51}$ \\
\hline
\end{tabular}

All costs were converted to 2020 USD using the medical care component of the Consumer Price Index.

mRS modified Rankin Scale; EVT, endovascular thrombectomy; mTICI, modified Thrombolysis in Cerebral Infarction; IVT, intravenous thrombolysis; HERMES, Highly Effective Reperfusion evaluated in Multiple Endovascular Stroke Trials; NIS, National Inpatient Sample.

*The minimum and maximum values for ranges were derived from reported 95\% confidence intervals or from calculated 95\% confidence intervals with the use of variance estimates as available.

costs for EVT and SC were added to the acute treatment costs within 90 days according to the specific strategy. Based on studies that investigated the material usage, procedure times and recanalization rates, we assumed that the EVT procedure costs are independent of patient age. ${ }^{30,31}$

The long-term annual treatment costs were based on contemporary data from the United States for each level of the $\mathrm{mRS}$ score. ${ }^{7}$ In line with the literature, the acute and long-term costs were not adjusted for patient age. ${ }^{22-29}$ All costs were converted to 2020 United States Dollars according to the medical care component of the Consumer Price Index ${ }^{43}$ and discounted by $3 \%$ each year. ${ }^{14}$

\section{Indirect costs}

To adopt a societal perspective, we assessed the indirect costs caused by childhood stroke based on the human capital ap- 
Score on modified Rankin Scale

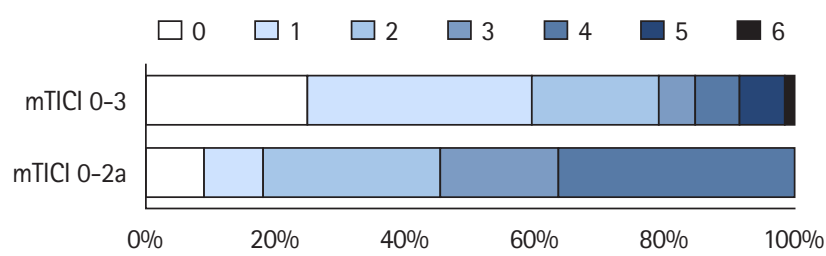

Figure 2. Save ChildS Study outcomes by recanalization grade. Pediatric stroke outcomes on the modified Rankin Scale during hospital discharge stratified by modified Thrombolysis in Cerebral Infarction (mTICl) scores. The $\mathrm{mTICl} 0-3$ group represents all patients in the Save ChildS study and was used to model outcomes of the thrombectomy group. The $\mathrm{mTICl} 0-2 \mathrm{a}$ group represents patients with unsuccessful recanalization; these outcomes were used to model the standard of care group.

proach. According to this approach, the amount of the productivity losses is measured based on (1) the lost productivity due to premature mortality in stroke patients; (2) the reduced productivity that is caused by the morbidity of stroke survivors; and (3) the costs for informal caregiving by next of kin. ${ }^{14}$

To analyze the costs caused by premature mortality (1), we used age-dependent productivity measures based on nationwide average gross wages of the employed population. Gross wage data were available for citizens from 16 years up to 80 years of age by the United States Census Bureau (Current Population Survey)..$^{44}$ These productivity measures were combined with the general population employment status provided by the United States Bureau of Labor Statistics. ${ }^{45}$ Non-stroke-related deaths were not considered for premature mortality.

To model the costs implied by the reduced productivity of stroke survivors (2), we multiplied the above-mentioned productivity measures by the relative earnings of stroke survivors compared to the non-stroke population (82.5\%). ${ }^{46}$ To our knowledge, there is no data that represent the relative earnings depending on the functional disability as measured on the mRS scale. As there is consensus on the fact that the patient's level of disability is the most important predictor of return-to-work (RTW), ${ }^{47}$ we used the functional outcome as an alternative approach as previously described. ${ }^{47}$ We multiplied each above-mentioned productivity measure by the RTW probability of stroke survivors, respective of their current level of disability and calculated the difference to the annual earnings of a healthy age-matched individual. We used reported RTW probabilities stratified by individual mRS health states ( $m R S ~ 0,0.63$; $m R S ~ 1,0.72 ; m R S ~ 2,0.49 ; m R S ~ 3,0.19 ; m R S ~ 4,0.14 ; m R S 5$, $0.00) .^{48}$ RTW was shown to be mainly influenced by the degree of disability than by patient age. ${ }^{49,50}$

The annual costs for informal caregiving (3) were based on estimates for the United States ${ }^{51}$ and stratified by degrees of
Table 2. Results of probabilistic sensitivity analyses

\begin{tabular}{|c|c|c|}
\hline Variable & Healthcare perspective & Societal perspective \\
\hline \multicolumn{3}{|l|}{ EVT strategy } \\
\hline Lifetime effectiveness & 20.32 OALYs & 20.34 QALYS \\
\hline Lifetime costs & $\$ 559,212$ & $\$ 888,782$ \\
\hline Lifetime NMB* & $\$ 455,029$ & $\$ 125,627$ \\
\hline \multicolumn{3}{|l|}{ SC strategy } \\
\hline Lifetime effectiveness & 16.33 OALYs & 16.32 OALYs \\
\hline Lifetime costs & $\$ 729,195$ & $\$ 1,142,892$ \\
\hline Lifetime NMB* & $\$ 85,219$ & $-\$ 326,351$ \\
\hline \multicolumn{3}{|l|}{ EVT compared to SC strategy } \\
\hline Incremental effectiveness & 3.99 OALYS & 4.02 OALYs \\
\hline Incremental costs & $-\$ 169,982$ & $-\$ 254,110$ \\
\hline Incremental $\mathrm{NMB}^{*}$ & $\$ 369,810$ & $\$ 451,978$ \\
\hline \multicolumn{3}{|l|}{ EVT acceptability at WTP } \\
\hline$\$ 50,000 / 0 A L Y$ & $96.08 \%$ & $96.61 \%$ \\
\hline$\$ 100,000 / 0 A L Y$ & $96.49 \%$ & $96.64 \%$ \\
\hline$\$ 150,000 / 0 A L Y$ & $96.60 \%$ & $96.66 \%$ \\
\hline
\end{tabular}

All values are median values derived from probabilistic sensitivity analyses using 10,000 second order Monte Carlo simulation runs.

EVT, endovascular thrombectomy; OALY, quality-adjusted life year; NMB, net monetary benefit; SC, standard of care; WTP, willingness-to-pay.

*The WTP threshold was set to $\$ 100,000 / 0 A L Y$ for all NMB calculations. Negative incremental costs indicate cost-savings and positive incremental NMB values represent higher net monetary benefits associated with EVT.

disability (mRS 0-1 vs. mRS 2-5). The hourly wages for home health aides in the United States of the recently reported year 2018 were used to calculate annual costs. These additional input parameters to model the societal perspective are provided in Table 1.

\section{Utilities}

Therapy effectiveness was measured by quality-adjusted life years (QALYs), calculated by multiplying years spent in mRS health states by assigned utility weights. Utility weights were derived from a recent survey that used a patient-centered approach. ${ }^{33}$ In line with several studies on health-related quality of life after ischemic stroke, patient age was assumed to have no significant influence on the utility weights. ${ }^{34-37}$ Values range from 0.0 to 1.0, with 0.0 representing no, and 1.0 representing perfect quality of life. All QALYs were discounted by $3 \%$ each year. $^{14}$

\section{Cost-effectiveness analysis}

Treatment strategies were compared in terms of incremental costs, incremental effectiveness and incremental cost-effectiveness ratios. In line with recent recommendations, ${ }^{14,52}$ we applied different willingness-to-pay (WTP) thresholds $(\$ 50,000$, 
$\$ 100,000$, and \$150,000 per QALY). Cost-effectiveness acceptability rates were determined for different patient ages.

\section{Sensitivity analysis}

To test the robustness of the model, we conducted probabilistic sensitivity analyses, allowing for simultaneous alteration of multiple model input parameters. The starting age was set to the median age in the Save ChildS Study of 11.3 years. Distribution types were used according to probability density functions for $2 n d$ order Monte Carlo simulation runs $(n=10,000)$. The distribution type for the model input parameters is shown in Table 1. To study the impact of the pediatric recurrent stroke rate in the first year after index stroke, deterministic one-way sensitivity analysis was performed.

\section{Results}

\section{Probabilistic sensitivity analyses}

The model results yielded EVT as the dominant (i.e., cost-effective as well as cost-saving) strategy for pediatric stroke patients. The median cumulative effectiveness over the lifetime was estimated with 20.33 OALYs for EVT treatment and 16.33 OALYS for SC treatment. The median lifetime healthcare costs amounted to $\$ 559,212$ for EVT treatment and to $\$ 729,195$ for SC treatment. The incremental effectiveness for the average age of 11.3 years at first stroke in the Save ChildS Study was thus determined as an additional 4.02 lifetime QALYs, with lifetime cost-savings that amounted to $\$ 169,982$ from a healthcare perspective and $\$ 254,110$ when applying a societal perspective.

Acceptability rates for EVT were consistently higher than 95\% for all tested WTP thresholds and for both perspectives. EVT reached $96.49 \%$ and $96.64 \%$ acceptability at a WTP of $\$ 100,000 /$ ALY for the healthcare and societal perspectives. The detailed results are presented in Table 2 . Cost-effectiveness planes showing scatter plots of incremental costs and effectiveness of the 10,000 simulation runs are presented in Figure 3.

\section{One-way sensitivity analysis of pediatric recur- rent stroke rate}

The deterministic one-way sensitivity analysis for first-year recurrent stroke rate yielded cost-effectiveness for EVT even up to a rate of $50 \%$. The lifetime costs remained lower with EVT across the range from $0 \%$ to 50\% (Figure 4A), while incremental QALYs continually declined as a result of the shortened life expectancy (Figure 4B). Incremental net monetary benefit values remained positive across the range from $0 \%$ up to $50 \%$ first-year recurrent stroke rate (Figure 4C).

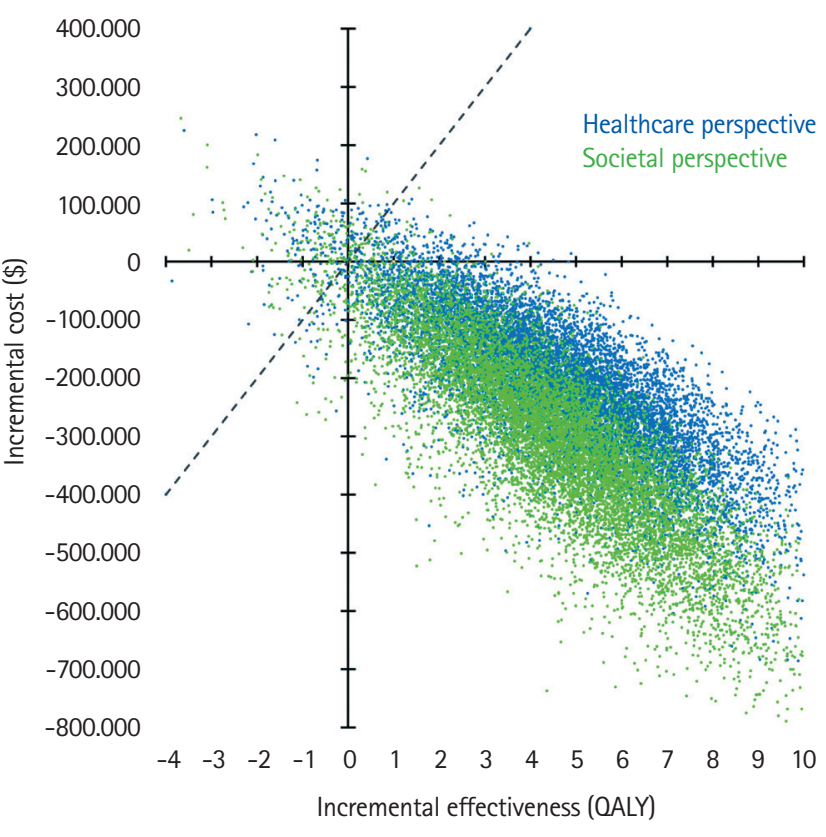

Figure 3. Probabilistic sensitivity analysis from the healthcare and societal perspective. Cost-effectiveness planes of incremental costs and incremental effectiveness of endovascular thrombectomy (EVT) versus standard care are shown for the probabilistic sensitivity analysis. Each dot represents one simulation run. The dashed line indicates a willingness-to-pay threshold of $\$ 100,000 / q u a l i t y-a d j u s t e d ~ l i f e$ year (QALY). Dots right to this line are considered cost-effective simulation runs. Dots in the right lower quadrant indicate cost-savings combined with incremental QALYs by EVT.

\section{Discussion}

Our study systematically investigated the cost-effectiveness of EVT for pediatric patients with LVO stroke. Adopting a United States healthcare as well as a societal perspective, EVT was the dominant strategy, resulting in significant lifetime health benefits and cost-savings compared with conventional SC management. From an economic point of view, there is clear support for EVT as the preferred treatment strategy in this patient population.

While several large prospective randomized trials have shown the clear benefit of EVT in adulthood stroke, making it the SC in acute LVO, only small cohorts have been published investigating the use of EVT in childhood stroke. ${ }^{11,53}$ The Save ChildS study was the first multicenter retrospective analysis of the use of EVT for LVO in children, demonstrating technical and clinical success rates comparable to the large RCTs in adults, with a good safety profile. This holds even true for children with LVO presenting in the late therapeutic window of up to 24 hours after onset of symptoms and different age groups. ${ }^{54,55}$

As prospective randomized trials on EVT in children will most likely never become available, the evidence level will be based on those retrospective cohorts as well as prospective registry 


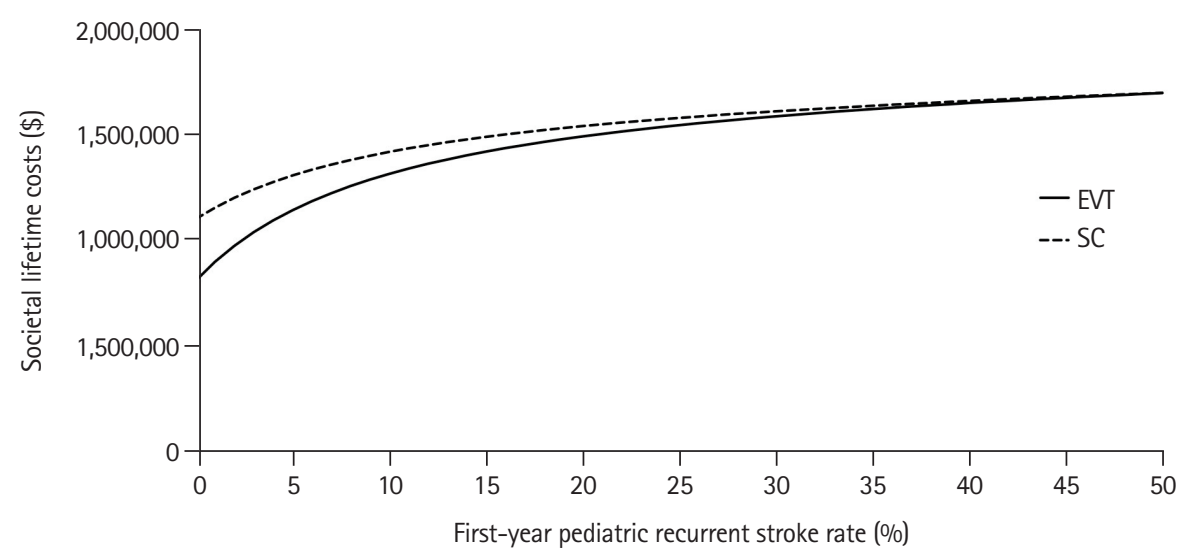

A

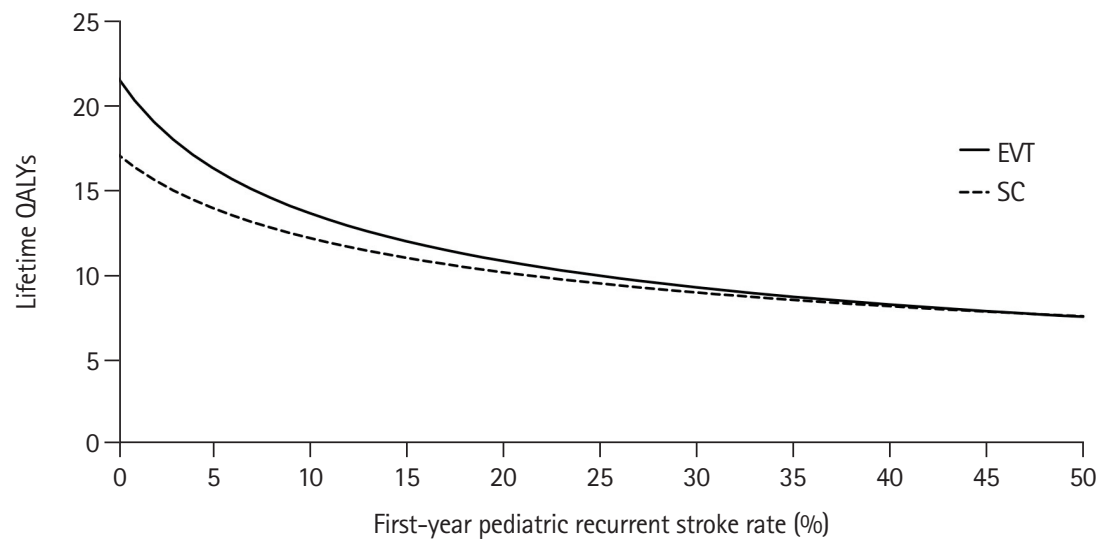

B

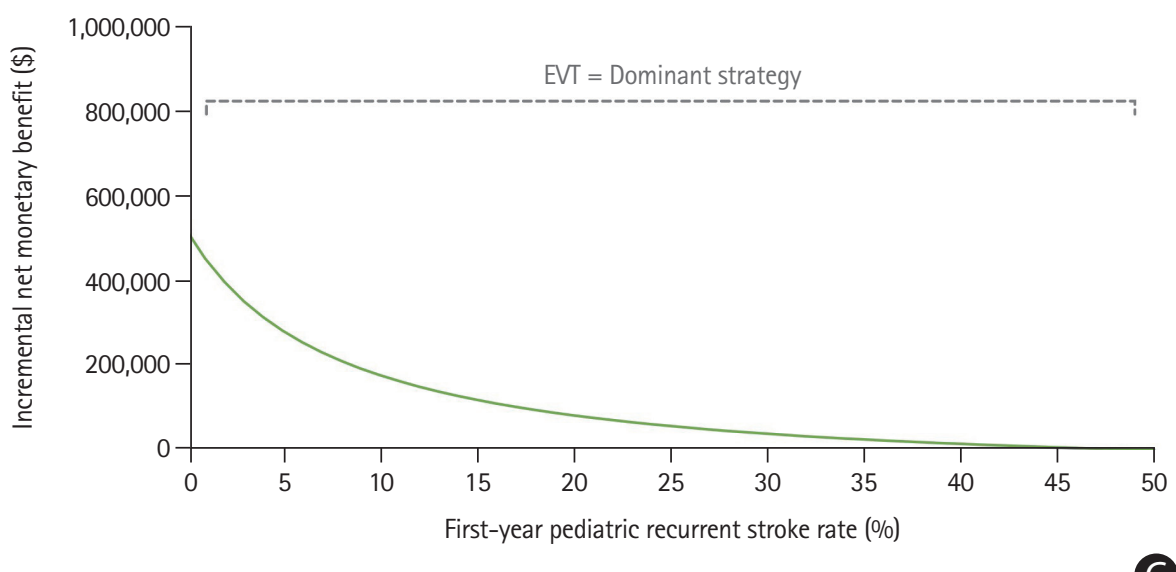

Figure 4. Sensitivity analysis of first-year pediatric recurrent stroke rate. Deterministic one-way sensitivity analysis results are shown for the societal perspective, including societal lifetime costs (A), lifetime quality-adjusted life years (OALYs) (B), and incremental net monetary benefit for endovascular thrombectomy (EVT) compared with standard care (SC) (C). Positive incremental net monetary benefit values indicate cost-effectiveness of EVT based on a willingness-to-pay of $\$ 100,000 / 0 A L Y$.

data. Even though there are several borderline indications such as underlying arteriopathies, ${ }^{56}$ in light of the moderate evidence of intravenous thrombolysis in pediatric LVO, ${ }^{9,10} \mathrm{EVT}$ is increasingly advocated in the pediatric stroke community. ${ }^{57,58}$

The major strength of this analysis is a detailed literature re- view regarding age dependency of model input parameters and the modification during the pediatric and adult timeline during the lifetime simulations as well as data access to the largest contemporary cohort on pediatric EVT. Moreover, this study included a healthcare as well as a societal perspective, as cur- 
rently recommended by the Second Panel on Cost-Effectiveness in Health and Medicine. ${ }^{14}$

There are several limitations of our study that need to be taken into account when interpreting the results. First, the study design was retrospective with the inherent limitations of this type of design, for example regarding model input data or change in procedure over time of recruitment. Second, outcomes for EVT and SC had to be modeled based on successful and futile recanalization as no randomized data or respective $\mathrm{SC}$ cohort data were available. As the complication rate of EVT is also very low in children, this approach may best model the differences in outcome and has been used before to study EVT treatment effects in large core strokes in adults. ${ }^{59}$ Third, it was not feasible to study different pediatric age subgroups based on the limited patient numbers. Fourth, several of the cost input parameters had to be modelled on the basis of adult data as there were no corresponding pediatric data available in the literature. Fifth, mRS was applied as the outcome parameter that defined the different health states. This was also due to a paucity of data on pediatric stroke outcome measures regarding modeling of quality of life and direct as well as indirect costs. Sixth, due to the lack of data for childhood stroke we had to assume that the RTW after stroke was mainly influenced by the mRS outcome, which was only available from a small cohort and had limited data for the mRS 0 subgroup. No data was available to model RTW changes during the process of aging.

\section{Conclusions}

In conclusion, our analysis of EVT based on the Save ChildS Study resulted in added QALYs and reduced lifetime costs. Based on the available data, EVT is very likely to be a cost-effective and cost-saving treatment strategy for childhood stroke.

\section{Disclosure}

Jens Fiehler reported serving as a paid consultant for Acandis, Boehringer Ingelheim, Cerenovus, Covidien, Evasc Neurovascular, MD Clinicals, Medtronic, Medina, Microvention, Penumbra, Route92, Stryker, and Transverse Medical. Jens Fiehler is also CEO of Eppdata. Dr Chapot reported receiving personal fees from Microvention, Stryker, Medtronic, Balt, Siemens, and Rapid Medical outside the submitted work. Franziska Dorn received personal fees for consultancy from Acandis, Phenox, Balt, and Cerenovus. Hans Henkes reported receiving personal fees and nonfinancial support from Phenox $\mathrm{GmbH}$ during the conduct of the study; in addition, Hans Henkes had patents issued to 10 2004040 868.8, 200580033578.6, 11/678,285, 502006012
111.5, PCT/EP 2005/ 009057, 05777 177.6-2318, and 102005 059670.3 , and is coinventor of several other patents related to thrombectomy. Martin Wiesmann reported receiving grants, personal fees, and nonfinancial support from Stryker Neurovascular; personal fees and nonfinancial support from Medtronic, Penumbra Inc, and Siemens Healthcare; and nonfinancial support from ab medica, Acandis, Asahi Intecc, Cerenovus, Kaneka Pharmaceuticals, Mentice $A B$, Microvention, and Phenox outside the submitted work. Markus Möhlenbruch reported receiving grants and nonfinancial support from Balt; personal fees from Medtronic; grants, personal fees, and nonfinancial support from MicroVention; and grants and personal fees from Stryker outside the submitted work. Alexander Radbruch reported receiving grants and personal fees from Guerbet and Bayer outside the submitted work. André Kemmling reported receiving personal fees from Penumbra, Stryker, and Phenox outside the submitted work. No industry support of the project was obtained.

\section{References}

1. Virani SS, Alonso A, Aparicio HJ, Benjamin EJ, Bittencourt MS, Callaway CW, et al. Heart disease and stroke statistics: 2021 update: a report from the American Heart Association. Circulation 2021;143:e254-e743.

2. Goyal M, Menon BK, van Zwam WH, Dippel DW, Mitchell PJ, Demchuk AM, et al. Endovascular thrombectomy after large-vessel ischaemic stroke: a meta-analysis of individual patient data from five randomised trials. Lancet 2016;387: 1723-1731.

3. Leppert MH, Campbell JD, Simpson JR, Burke JF. Cost-effectiveness of intra-arterial treatment as an adjunct to intravenous tissue-type plasminogen activator for acute ischemic stroke. Stroke 2015;46:1870-1876.

4. Ganesalingam J, Pizzo E, Morris S, Sunderland T, Ames D, Lobotesis K. Cost-utility analysis of mechanical thrombectomy using stent retrievers in acute ischemic stroke. Stroke 2015; 46:2591-2598.

5. Aronsson $M$, Persson J, Blomstrand $C$, Wester $P$, Levin LÅ. Cost-effectiveness of endovascular thrombectomy in patients with acute ischemic stroke. Neurology 2016;86:1053-1059.

6. Kunz WG, Hunink MG, Sommer WH, Beyer SE, Meinel FG, Dorn $F$, et al. Cost-effectiveness of endovascular stroke therapy: a patient subgroup analysis from a US healthcare perspective. Stroke 2016;47:2797-2804.

7. Shireman TI, Wang K, Saver JL, Goyal M, Bonafé A, Diener HC, et al. Cost-effectiveness of solitaire stent retriever thrombectomy for acute ischemic stroke: results from the SWIFT-PRIME 
trial (Solitaire With the Intention for Thrombectomy as Primary Endovascular Treatment for Acute Ischemic Stroke). Stroke 2017;48:379-387.

8. Jordan LC, Hillis AE. Challenges in the diagnosis and treatment of pediatric stroke. Nat Rev Neurol 2011;7:199-208.

9. Rivkin MJ, deVeber G, Ichord RN, Kirton A, Chan AK, Hovinga $\mathrm{CA}$, et al. Thrombolysis in pediatric stroke study. Stroke 2015;46:880-885.

10. Amlie-Lefond C, Shaw DW, Cooper A, Wainwright MS, Kirton $A$, Felling $R J$, et al. Risk of intracranial hemorrhage following intravenous tPA (tissue-type plasminogen activator) for acute stroke is low in children. Stroke 2020;51:542-548.

11. Sporns PB, Kemmling A, Hanning U, Minnerup J, Sträter R, Niederstadt T, et al. Thrombectomy in Childhood Stroke. J Am Heart Assoc 2019;8:e011335.

12. Sporns PB, Sträter R, Minnerup J, Wiendl H, Hanning U, Chapot $R$, et al. Feasibility, safety, and outcome of endovascular recanalization in childhood stroke: the Save ChildS Study. JAMA Neurol 2020;77:25-34.

13. Ellis C, McGrattan $K$, Mauldin P, Ovbiagele B. Costs of pediatric stroke care in the United States: a systematic and contemporary review. Expert Rev Pharmacoecon Outcomes Res 2014;14:643-650.

14. Sanders GD, Neumann PJ, Basu A, Brock DW, Feeny D, Krahn $M$, et al. Recommendations for conduct, methodological practices, and reporting of cost-effectiveness analyses: Second Panel on Cost-Effectiveness in Health and Medicine. JAMA 2016;316:1093-1103.

15. Luengo-Fernandez R, Paul NL, Gray AM, Pendlebury ST, Bull LM, Welch SJ, et al. Population-based study of disability and institutionalization after transient ischemic attack and stroke: 10-year results of the Oxford Vascular Study. Stroke 2013;44:2854-2861.

16. Wolfe CD, Crichton SL, Heuschmann PU, McKevitt CJ, Toschke AM, Grieve AP, et al. Estimates of outcomes up to ten years after stroke: analysis from the prospective South London Stroke Register. PLoS Med 2011;8:e1001033.

17. Ganesh A, Luengo-Fernandez $R$, Wharton RM, Gutnikov SA, Silver $L E$, Mehta $Z$, et al. Time course of evolution of disability and cause-specific mortality after ischemic stroke: implications for trial design. J Am Heart Assoc 2017;6:e005788.

18. Pennlert J, Eriksson M, Carlberg B, Wiklund PG. Long-term risk and predictors of recurrent stroke beyond the acute phase. Stroke 2014;45:1839-1841.

19. Arias $E_{1} \mathrm{Xu}$ J. United States life tables, 2018. Natl Vital Stat Rep 2020;69:1-45.

20. Slot KB, Berge $E$, Sandercock $P$, Lewis SC, Dorman $P$, Dennis $M$, et al. Causes of death by level of dependency at 6 months after ischemic stroke in 3 large cohorts. Stroke 2009;40:1585-1589.

21. Dawson J, Lees JS, Chang TP, Walters MR, Ali M, Davis SM, et al. Association between disability measures and healthcare costs after initial treatment for acute stroke. Stroke 2007; 38:1893-1898.

22. Caro JJ, Huybrechts KF, Duchesne I. Management patterns and costs of acute ischemic stroke: an international study. For the Stroke Economic Analysis Group. Stroke 2000;31:582-590.

23. Caro JJ, Huybrechts KF, Kelley HE. Predicting treatment costs after acute ischemic stroke on the basis of patient characteristics at presentation and early dysfunction. Stroke 2001; 32:100-106.

24. Luengo-Fernandez R, Gray AM, Rothwell PM. Population-based study of determinants of initial secondary care costs of acute stroke in the United Kingdom. Stroke 2006; 37:2579-2587.

25. Luengo-Fernandez R, Gray AM, Rothwell PM. Effect of urgent treatment for transient ischaemic attack and minor stroke on disability and hospital costs (EXPRESS study): a prospective population-based sequential comparison. Lancet Neurol 2009;8:235-243.

26. Yoneda Y, Uehara T, Yamasaki H, Kita Y, Tabuchi M, Mori E. Hospital-based study of the care and cost of acute ischemic stroke in Japan. Stroke 2003;34:718-724.

27. Diringer MN, Edwards DF, Mattson DT, Akins PT, Sheedy CW, $\mathrm{Hsu} \mathrm{CY}$, et al. Predictors of acute hospital costs for treatment of ischemic stroke in an academic center. Stroke 1999;30:724728.

28. Alvarez-Sabín J, Quintana M, Masjuan J, Oliva-Moreno J, Mar J, Gonzalez-Rojas N, et al. Economic impact of patients admitted to stroke units in Spain. Eur J Health Econ 2017;18: 449-458.

29. Holloway RG, Witter DM Jr, Lawton KB, Lipscomb J, Samsa G. Inpatient costs of specific cerebrovascular events at five academic medical centers. Neurology 1996;46:854-860.

30. Castonguay AC, Zaidat 00, Novakovic R, Nguyen TN, Taqi MA, Gupta $R$, et al. Influence of age on clinical and revascularization outcomes in the North American Solitaire Stent-Retriever Acute Stroke Registry. Stroke 2014;45:3631-3636.

31. Beumer D, Rozeman AD, Lycklama À Nijeholt GJ, Brouwer PA, Jenniskens SF, Algra $A$, et al. The effect of age on outcome after intra-arterial treatment in acute ischemic stroke: a MR CLEAN pretrial study. BMC Neurol 2016;16:68.

32. Chambers MG, Koch P, Hutton J. Development of a decision-analytic model of stroke care in the United States and Europe. Value Health 2002;5:82-97.

33. Chaisinanunkul N, Adeoye O, Lewis RJ, Grotta JC, Broderick J, Jovin TG, et al. Adopting a patient-centered approach to prima- 
ry outcome analysis of acute stroke trials using a utilityweighted modified Rankin scale. Stroke 2015;46:2238-2243.

34. Hallan S, Asberg A, Indredavik B, Widerøe TE. Quality of life after cerebrovascular stroke: a systematic study of patients' preferences for different functional outcomes. J Intern Med 1999;246:309-316.

35. Gargano JW, Reeves MJ; Paul Coverdell National Acute Stroke Registry Michigan Prototype Investigators. Sex differences in stroke recovery and stroke-specific quality of life: results from a statewide stroke registry. Stroke 2007;38:25412548.

36. Xie J, Wu EQ, Zheng ZJ, Croft JB, Greenlund KJ, Mensah GA, et al. Impact of stroke on health-related quality of life in the noninstitutionalized population in the United States. Stroke 2006;37:2567-2572.

37. Naess $H$, Waje-Andreassen $U$, Thomassen $L$, Nyland $H$, Myhr KM. Health-related quality of life among young adults with ischemic stroke on long-term follow-up. Stroke 2006;37: 1232-1236.

38. Kunz WG, Hunink MG, Dimitriadis $K$, Huber $T$, Dorn F, Meinel $F G$, et al. Cost-effectiveness of endovascular therapy for acute ischemic stroke: a systematic review of the impact of patient age. Radiology 2018;288:518-526.

39. Sträter $R$, Becker $S$, von Eckardstein $A$, Heinecke $A$, Gutsche $S$, Junker $R$, et al. Prospective assessment of risk factors for recurrent stroke during childhood: a 5-year follow-up study. Lancet 2002;360:1540-1545.

40. Hillen $T$, Coshall $C$, Tilling $K$, Rudd AG, McGovern R, Wolfe $C D$, et al. Cause of stroke recurrence is multifactorial: patterns, risk factors, and outcomes of stroke recurrence in the South London Stroke Register. Stroke 2003;34:1457-1463.

41. Hardie K, Hankey GJ, Jamrozik K, Broadhurst RJ, Anderson C. Ten-year risk of first recurrent stroke and disability after first-ever stroke in the Perth Community Stroke Study. Stroke 2004;35:731-735.

42. Mohan KM, Wolfe CD, Rudd AG, Heuschmann PU, Kolominsky-Rabas PL, Grieve AP. Risk and cumulative risk of stroke recurrence: a systematic review and meta-analysis. Stroke 2011;42:1489-1494.

43. Consumer Price Index. U.S. Bureau of Labor Statistics. http:// www.bls.gov/cpi. 2020. Accessed October 28, 2021.

44. Current population survey. United States Census Bureau. https://www.census.gov/cps/data/cpstablecreator.html. 2019. Accessed October 28, 2021.

45. Household data annual averages: 3. employment status of the civilian noninstitutional population by age, sex, and race. U.S. Bureau of Labor Statistics. https://www.bls.gov/cps/ cpsaat03.pdf. 2020. Accessed October 28, 2021.
46. Vyas MV, Hackam DG, Silver FL, Laporte A, Kapral MK. Lost productivity in stroke survivors: an econometrics analysis. Neuroepidemiology 2016;47:164-170.

47. Brown DL, Boden-Albala $B$, Langa KM, Lisabeth LD, Fair M, Smith MA, et al. Projected costs of ischemic stroke in the United States. Neurology 2006;67:1390-1395.

48. Tanaka $H$, Toyonaga $T$, Hashimoto $H$. Functional and occupational characteristics predictive of a return to work within 18 months after stroke in Japan: implications for rehabilitation. Int Arch Occup Environ Health 2014;87:445-453.

49. Saeki S, Ogata H, Okubo T, Takahashi K, Hoshuyama T. Factors influencing return to work after stroke in Japan. Stroke 1993;24:1182-1185.

50. Ferro JM, Crespo M. Prognosis after transient ischemic attack and ischemic stroke in young adults. Stroke 1994;25:16111616.

51. Hickenbottom SL, Fendrick AM, Kutcher JS, Kabeto MU, Katz SJ, Langa KM. A national study of the quantity and cost of informal caregiving for the elderly with stroke. Neurology 2002;58:1754-1759.

52. Neumann PJ, Cohen JT, Weinstein MC. Updating cost-effectiveness: the curious resilience of the $\$ 50,000$-per-QALY threshold. N Engl J Med 2014;371:796-797.

53. Bigi S, Dulcey A, Gralla J, Bernasconi C, Melliger A, Datta AN, et al. Feasibility, safety, and outcome of recanalization treatment in childhood stroke. Ann Neurol 2018;83:1125-1132.

54. Sporns PB, Psychogios MN, Straeter R, Hanning U, Minnerup J, Chapot $R$, et al. Clinical diffusion mismatch to select pediatric patients for embolectomy 6 to 24 hours after stroke: an analysis of the Save ChildS Study. Neurology 2021;96:e343-e351.

55. Sporns PB, Straeter R, Minnerup J, Wiendl H, Hanning U, Chapot $R$, et al. Does device selection impact recanalization rate and neurological outcome?: an analysis of the Save ChildS Study. Stroke 2020;51:1182-1189.

56. Sun LR, Harrar D, Drocton G, Castillo-Pinto C, Felling R, Carpenter $\mathrm{J}$, et al. Mechanical thrombectomy for acute ischemic stroke: considerations in children. Stroke 2020;51:3174-3181.

57. Chabrier S, Ozanne A, Naggara O, Boulouis G, Husson B, Kossorotoff M. Hyperacute recanalization strategies and childhood stroke in the evidence age. Stroke 2021;52:381-384.

58. Sporns PB, Psychogios M. Thrombectomy in childhood stroke: important considerations in borderline indications. Stroke 2020;51:2890-2891.

59. Broocks G, Hanning U, Flottmann F, Schönfeld M, Faizy TD, Sporns $\mathrm{P}$, et al. Clinical benefit of thrombectomy in stroke patients with low ASPECTS is mediated by oedema reduction. Brain 2019;142:1399-1407. 